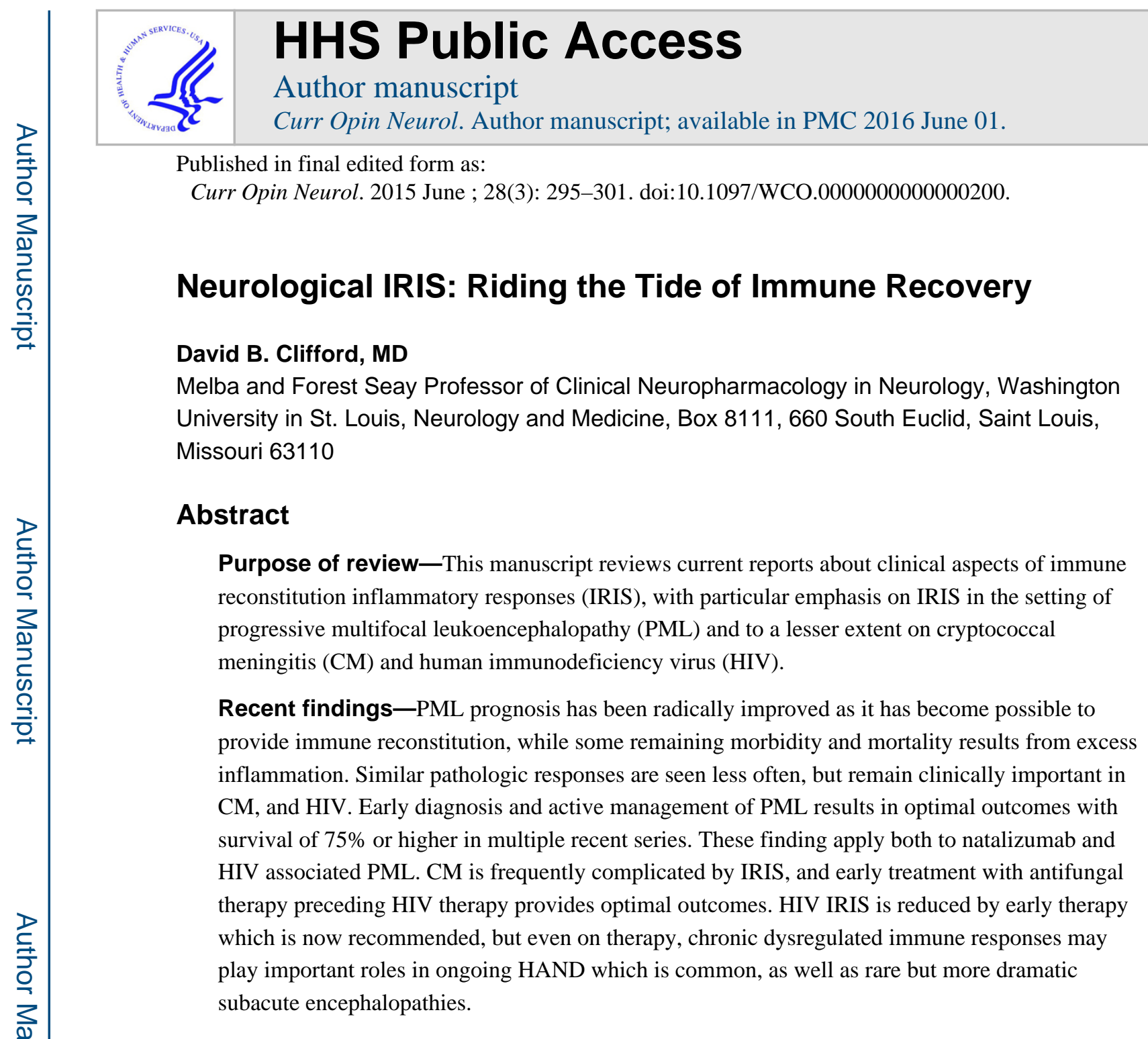

Summary-The clinician must actively monitor and treat both OI and the inflammatory response that is essential to recovery but may itself augment pathology and injury.

\title{
Introduction
}

One of the dynamic topics in neurology that is particularly relevant to infectious disease is the expanding knowledge about immunology and the nervous system. The blood brain barrier (BBB) is a complex physical and biological entity, closely regulating the environment in the brain. The HIV epidemic has provided unique opportunities to observe the consequences of long term cellular immunodeficiency in large numbers of individuals, while development of successful combined antiretroviral therapy (cART) has resulted in routine functional reversal of this immunodeficiency. Several opportunistic infections (OI) of the CNS occur frequently in this setting. These observations are now combined with experience from use of immune modulating therapies that have proven valuable treating autoimmune and neoplastic processes, but again result in unique oscillation of the immune status of the patients in whom they are used. The most intensively monitored such experience has been that of natalizumab, an integrin inhibiting monoclonal antibody, that

Telephone 314-747-8423, cliffordd@wustl.edu. 
has reduced relapse in many patients with relapsing-remitting multiple sclerosis, but also has a serious risk of progressive multifocal leukoencephalopathy (PML). This opportunistic brain infection almost exclusively is seen in settings of chronic cellular immunodeficiency. While natalizumab which does not broadly compromise immunity, it limits immune surveillance in the brain and may induce seeding of brain with JC virus. In addition, with chemotherapy for a variety of neoplastic or inflammatory conditions, the recovery of the immune response after severe immunodeficiency occurs with cycles of therapy, providing yet other situations in which fluctuating immunodeficiency is generated in the modern practice of medicine. This review will update the reader on recent observations of importance to neurology associated with immune reconstitution inflammatory syndromes (IRIS) focusing on PML, cryptococcal meningitis and HIV encephalitis. Space will not allow discussion of other opportunistic infections (OI) that also demonstrate abnormal inflammatory responses, including tuberculosis, varicella and toxoplasmosis. ${ }^{1-6}$

\section{Immune Reconstitution Inflammatory Syndrome (IRIS)}

Recognition of clinically important IRIS emerged along with the advent of cART to treat HIV in the later 1990s. ${ }^{7}$ In the setting of HIV, as successful treatment is accomplished, HIV viral loads fall and CD4 lymphocyte counts rise, yet paradoxically the patient may experience clinical deterioration. On closer examination this deterioration is driven by a robust inflammatory response rather than by underlying HIV driven pathophysiology. Although onset may be delayed, IRIS most commonly occurs in the initial few months after initiation of cART, demonstrating the prompt re-emergence of functional immune surveillance with cART. Most commonly, the paradoxical inflammation is directed at an opportunistic pathogen or tumor. This manuscript will focus on neurological IRIS in the setting of progressive multifocal leukoencephalopathy (PML), cryptococcal meningitis (CM), or HIV encephalitis(HIVE) where this phenomenon has had greatest impact. A more exhaustive review of neurological IRIS has recently been published by Post et al. ${ }^{1,5}$ It is routinely noted that IRIS is more likely to occur and may be more intense when the precipitating immune deficiency state has been severe and prolonged, and when immune reconstitution is most effective. IRIS is a particularly salient topic in developing world settings since HIV disease may be treated late, and opportunistic infections more often precipitate the initiation of HIV therapy. ${ }^{8}$

\section{IRIS Can Be Classic or Unmasking}

The classic presentation of IRIS occurs when an opportunistic infection (OI) is noted first with its associated symptoms. When immune reconstitution is accomplished as part of the therapy for the opportunistic pathogen, worsening of OI symptoms occurs driven by inflammation rather than the underlying disease. In a recent review from one center using clinically defined IRIS, the incidence of IRIS was $>10 \%$ on for Kaposi sarcoma, tuberculosis or Cryptococcus. ${ }^{9}$ However, such clinical observations likely underestimate IRIS because it may be difficult to differentiate aspects of IRIS from that of underlying OI.

Sometimes however, immune reconstitution occurs when the underlying pathogen is still asymptomatic. The inflammatory response to undiscovered pathogens makes them symptomatic, thus presenting as an "unmasking" of the OI in the setting of immune 
reconstitution. Earlier in the HIV epidemic, and in developing world settings, this is a frequent phenomenon as HIV treatment is started late. As early treatment of HIV becomes routine, such events are declining in frequency, and already recent reviews suggest that unmasking of OI is rather uncommon in experience with HIV in the developed world. ${ }^{10}$ It has also been seen on withdrawal of natalizumab treated multiple sclerosis (MS) prior to recognized PML diagnosis during natural recovery of immune surveillance. ${ }^{11}$

\section{Progressive Multifocal Leukoencephalopathy (PML) and IRIS}

PML has been a particularly dramatic setting in which IRIS has transformed the pathology and the prognosis of this dangerous disease. It is interesting that while the fundamental situation causing HIV or natalizumab associated PML differ radiacally, the clinical and pathological lessons are similar regardless of the underlying setting in which JC virus is transformed and invades the brain.

\section{Pathologic Observations of PML and IRIS}

Historically PML presented in very advanced immune deficient states, and was notable for lack of inflammatory response in the pathologic lesions. The BBB remained intact resulting in little contrast enhancement on gadolinium enhanced MRI. As immune competence is restored, PML lesions are transformed so that they are radically different pathologically, demonstrating substantial infiltrates of lymphocytes, many perivascular, resulting in damage to the $\mathrm{BBB}$, and development of MRI contrast enhancement and brain edema with mass effect. (Figure 1) Prognosis for survival of PML is optimized when effective immune competence is achieved but remains less than that causing massive tissue destruction or brain herniation. ${ }^{12}$ Effective therapy for PML by achieving immune reconstitution has resulted in survival as $70-80 \%$ in both HIV and natalizumab associated PML. ${ }^{13-15}$

Pathology and its correlates with clinical and imaging findings are too rarely synthesized. An important description of PML IRIS was reported by Huang. ${ }^{16}$ This report demonstrated inflammatory PML across several settings in which PML is observed, and crucially showed that MRI contrast enhancement was not sensitive to milder inflammatory PML. Careful pathologic characterization of the inflammatory responses in situ have demonstrated prevalence of cytotoxic CD8+ cells with the ratio of CD8+ cells to JCV infected cells seventy times higher in cases of IRIS than in those without inflammatory response. ${ }^{17}$ This cellular immune response is presumed to both contribute to control of JC infection and to tissue damage. Pathologic correlation in natalizumab associated PML where biopsies were performed similarly emphasized the invasion of CD8+ cells as well as plasma cells with clearance of viral laden cells. ${ }^{18}$ A recent post mortem report illustrates PML with IRIS in MS. It has been hypothesized that JC virus laden cells might enter the brain along with the MS associated inflammation. If this were the case, PML might preferentially occur near MS lesions. This manuscript demonstrates the PML lesions populated with CD4+ and CD8+ cells consistent with IRIS, were distinct from MS lesions. ${ }^{19}$ Pathologic studies also have helped to expand the spectrum of JC virus associated diseases, including allowing documentation of cerebellar granule cell neuronopathy which is seen in chemotherapy, HIV and natalizumab associated JC virus disease and may demonstrate IRIS. ${ }^{20-23}$ 


\section{Brain Imaging and PML-IRIS}

When paradoxical clinical deterioration occurs most clinicians seek to substantiate IRIS by demonstration of new contrast enhancement in PML lesions consistent with inflammatory breakdown of the BBB. While presence of enhancement is consistent with IRIS, it is not sensitive for it. Thus, absence of enhancement should not preclude clinical consideration of IRIS. Yousry and Wattjes have provided important MRI descriptions of natalizumab PML which is commonly inflammatory. ${ }^{24,25}$ Recognition of fundamental imaging patterns of PML, but with punctuate or linear enhancement is notable consistent with inflammatory PML most common in this setting. Development of mass effect is typical only of very advanced PML IRIS. The association of punctuate pattern consistent with perivascular inflammation in natalizumab associated PML was reiterated by Wattjes and likely is an early manifestation of PML with IRIS. ${ }^{26}$

Khoury et $\mathrm{al}^{27}$ recently emphasized a unique imaging finding of a hyperintense cortical signal observed in T1 uncontrasted MRI images of PML patients. This was very commonly associated with seizures, as well as being more common in the setting of IRIS. It is notable that descriptions of seizures associated with PML have increased since cART was available for HIV, and are quite common in natalizumab associated PML where there is more notable IRIS. A similar finding was also noted on imaging by Wattjes described as cortical laminar necrosis. ${ }^{25}$

Khoury et $\mathrm{al}^{27}$ have also recently reported application of arterial spin labeling MRI to assess perfusion in PML lesions. This preliminary study based on clinical diagnosis of IRIS suggested that marked hyperperfusion is seen in PML lesions, but not in those with ongoing IRIS. Additionally, use of proton magnetic resonance spectroscopy ( $\mathrm{H}^{1} \mathrm{MRS}$ ) has been reported to demonstrate elevated myoinositol and lipid/creatine peaks in lesions with inflammation. ${ }^{28,29}$ These observations bear validation, since means of determining IRIS and thus optimal therapeutic approach are needed.

\section{Therapy and Outcomes for PML IRIS}

PML has earned a reputation as a lethal and untreatable neurological complication that will almost invariably lead to death in six months or less. This reputation was deserved when almost all cases were seen in advanced AIDS or terminal malignancy patients. However, when immune suppression can be modified, the outlook for PML patients is improved. Survival is frequent, although the PML itself will leave deficits that vary from minor to severe. Mefloquine was the latest therapy tested to control JC infection, but a recent international study failed to demonstrate clinically useful activity in a trial dominated by HIV PML patients with a relatively good intrinsic outcome associated with cART. ${ }^{14}$ While effective antiviral therapy for JC remains an important goal, early diagnosis and optimal management of an effective immune reconstitution are critical clinical endeavors. ${ }^{12}$ Systematic active management of PML, including early diagnosis, rapid immune reconstitution, and management of IRIS and of seizures can result in $100 \%$ survival in small single site series, albeit with significant neurological complications. ${ }^{30}$ Aggressive HIV management can result in > 80\% survival for 6 months. ${ }^{13}$ The value of early diagnosis has been emphasized by excellent survival statistics when the PML develops after natalizumab 
withdrawal or is discovered by MRI prior to symptom onset. ${ }^{31}$ Dong-Si found that $96 \%$ of those natalizumab PML patients diagnosed presymptomaticaly survived versus $75 \%$ in those after PML symptoms had occurred, and the quality of outcomes was also superior. Fine et $\mathrm{al}^{11}$ described uniform survival of 15 PML patients with the disease emerging after natalizumab discontinuation representing primarily IRIS unmasking PML.

Rapid immune reconstitution appears necessary, and for natalizumab associated PML use of plasma exchange to speed this process has become common. ${ }^{32}$ While not supported by randomized trials, since natalizumab remains active for several months, it is rational to seek earlier immune recovery to optimize outcomes against this aggressive disease. However, there is little doubt that some patients actually die from the severity of IRIS. Thus active management of IRIS has been advocated, generally with high dose corticosteroid therapy. Current improved clinical outcomes have generally been achieved with substantial corticosteroid interventions. Thus, the theoretical danger of this seemingly irrational immunosuppressive therapy in the face of an OI should not preclude its practice. However, careful analysis of the impact of corticosteroids on JCV-specific T-cell responses, including significant decrease frequency of interferon- $\gamma$ and TNF-a producing JCV-specific CD8+ cells, emphasize reason for caution in starting corticosteroids prior to initiation of immune reconstitution. ${ }^{33}$ An interesting steroid sparing approach has been to block CCR5 mediated inflammation with the CCR5 antagonist antiretroviral maraviroc. Several groups have reported use of this antiretroviral drug in MS patients where it may contribute to blunting IRIS complications. ${ }^{34}$

While control of IRIS is generally the therapeutic objective in natalizumab associated PML, occasionally enhancing the immune response is required to treat PML where immune reconstitution is inadequate or delayed. In that setting, the role of interleukin 7 is presently being assessed in an HIV associated PML trial in France. Its use in conjunction with JC virus capsid vaccination to optimize the immune response to PML was recently reported. ${ }^{35}$

\section{IRIS in Cryptococcal Meningitis (CM)}

$\mathrm{CM}$ is a very common OI worldwide, and one in which IRIS critically modifies the therapeutic approach. ${ }^{9}$ Early diagnosis and treatment is important. Outcomes are worse when HIV therapy is initiated with CM therapy, rather than delaying until CM is well underway. In a recent clinical trial comparing early versus later cART initiation, deferring cART for 5 weeks after diagnosis and treatment of CM was associated with improved survival. ${ }^{36}$ Presymptomatic treatment of CM therapy is facilitated by emergence of lateral flow assay that can be economically done at the bedside in resource limited settings, and is increasing recommended prior to cART. ${ }^{36-44}$ Prediction of IRIS is associated with minimal pretreatment immune responses as manifest by cryptococcal mannoprotein activated CD4 T cell IFN- $\gamma$ responses ${ }^{45}$ or pre-treatment pro-inflammatory cytokines. ${ }^{46}$ The biology of CM and IRIS is likely to also be a function of the status of the polysaccharide capsule which contributes greatly to the immune response and is dynamically controlled. ${ }^{47,48}$ Treatment for CM IRIS includes efforts to remove the antigen by large volume lumbar punctures, as well as using corticosteroids if necessary. It is clear that IRIS may have a negative impact on 
outcomes for $\mathrm{CM}$ and thus successfully avoiding or minimizing it is an ongoing goal for successful therapy. ${ }^{49}$

\section{IRIS in HIV}

HIV has provided a workshop for learning about various manifestations of IRIS, most commonly when there are identified OI. However, there are reports of pathologic inflammatory responses absent identified pathogens save for HIV itself. Indeed, one of the leading hypotheses for HIV Associated Neurocognitive Disease (HAND) is that ongoing CNS inflammation leads to dysfunction due to long term toxicity of chronic inflammatory disease ${ }^{50,51}$ However, sometimes the inflammation takes a more aggressive clinical course, and results in progressive subacute deficits requiring the combination of ARV and immune suppressant drugs. ${ }^{52-54} \mathrm{~A}$ serious, rare syndrome has been reported in treated HIV patients with severe CD8+ infiltration of the brain that was fatal in $40 \%$ of the 10 cases reported, and appears to by a form of HIV associated IRIS. ${ }^{55}$ The pathologic trigger for IRIS response in this setting will require close assessment, but early attention on the HIV-Tat protein appears to provide a promising lead. ${ }^{56}$ Thus, the setting of HIV will continue to provide a wide spectrum of immune mediated conditions that may inform us further on both normal and pathologic control of the immune environment in the brain.

\section{Conclusion}

Immune modulation is transforming the approach to treatment of diseases as well as the opportunity to optimize treatment when opportunistic diseases occur. Outcomes for PML, cryptococcal meningitis and HIV all appear to be optimized by early detection and treatment. It is important to recognize that a dysregulated immune response often occurs in these settings, and that the optimal outcomes are likely to be achieved when the immune response can be augmented to control the OI without causing collateral tissue damage. Better ways of quantifying the degree and focus of the immune response, and expanding the arsenal of immune modulating therapies will achieve the best clinical outcomes.

\section{Acknowledgements}

Dr. Clifford has received support for consulting/advisory boards from Sanofi, Genentech, Quintiles, Inhibikase, BMS, GlaxoSmithKline, Millennium, Biogen Idec, Amgen, Pfizer, AstraZeneca, Cytheris and Merck. In addition, Dr. Clifford has received speaker honorarium from Sun Pharmaceuticals and Biogen Japan. Dr. Clifford receives research support from Lilly, Roche, Bavarian Nordic, Gilead, Alzheimer Association and NIH: NIAID, NIMH, NINDS, NIA and NINR. The author particularly thanks the contributions of many patients who have inspired and taught him regarding these conditions, as well as referring doctors who have shared their challenging patients with Dr. Clifford.

Funding: none for this work

\section{References}

1. Post MJ, Thurnher MM, Clifford DB, et al. CNS-immune reconstitution inflammatory syndrome in the setting of HIV infection, part 2: discussion of neuro-immune reconstitution inflammatory syndrome with and without other pathogens. AJNR American journal of neuroradiology. 2013; 34:1308-18. *Lengthy detailed review with emphasis on imaging IRIS. [PubMed: 22790252] 
2. Laureillard D, Marcy O, Madec Y, et al. Paradoxical tuberculosis-associated immune reconstitution inflammatory syndrome after early initiation of antiretroviral therapy in a randomized clinical trial. AIDS. 2013; 27:2577-86. [PubMed: 24096631]

3. Lagier JC, Raoult D. Immune reconstitution inflammatory syndrome associated with bacterial infections. Expert Opin Drug Saf. 2014; 13:341-50. [PubMed: 24512544]

4. Luetkemeyer AF, Kendall MA, Nyirenda M, et al. Tuberculosis immune reconstitution inflammatory syndrome in A5221 STRIDE: timing, severity, and implications for HIV-TB programs. J Acquir Immune Defic Syndr. 2014; 65:423-8. [PubMed: 24226057]

5. Post MJ, Thurnher MM, Clifford DB, et al. CNS-immune reconstitution inflammatory syndrome in the setting of HIV infection, part 1: overview and discussion of progressive multifocal leukoencephalopathy-immune reconstitution inflammatory syndrome and cryptococcal-immune reconstitution inflammatory syndrome. AJNR American journal of neuroradiology. 2013; 34:1297307. **Review of HIV associated IRIS relevant to the nervous system. [PubMed: 22790246]

6. Iro MA, Kirkham FJ, Macdonald JH, Tebruegge M, Faust SN, Patel SV. Varicella zoster virus central nervous system immune reconstitution inflammatory syndrome presenting in a child. The Pediatric infectious disease journal. 2013; 32:1283-4. [PubMed: 24141801]

7. French MA, Price P, Stone SF. Immune restoration disease after antiretroviral therapy. AIDS. 2004; 18:1615-27. [PubMed: 15280772]

8. Mateen FJ, Nath A. Central nervous system-immune reconstitution inflammatory syndrome in resource-limited settings: current burden and future needs. AIDS. 2012; 26:1851-5. [PubMed: 22781220]

9. Achenbach CJ, Harrington RD, Dhanireddy S, Crane HM, Casper C, Kitahata MM. Paradoxical immune reconstitution inflammatory syndrome in HIV-infected patients treated with combination antiretroviral therapy after AIDS-defining opportunistic infection. Clinical infectious diseases : an official publication of the Infectious Diseases Society of America. 2012; 54:424-33. [PubMed: 22095568]

10. Opportunistic infections and AIDS malignancies early after initiating combination antiretroviral therapy in high-income countries. AIDS. 2014; 28:2461-73. [PubMed: 25265230]

11. Fine AJ, Sorbello A, Kortepeter C, Scarazzini L. Progressive multifocal leukoencephalopathy after natalizumab discontinuation. Ann Neurol. 2014; 75:108-15. [PubMed: 24242357]

12. Clifford DB. Progressive multifocal leukoencephalopathy therapy. J Neurovirol. $2014 * *$ Review of therapeutics including management of IRIS for treatment of PML.

13. Gasnault J, Costagliola D, Hendel-Chavez H, et al. Improved survival of HIV-1-infected patients with progressive multifocal leukoencephalopathy receiving early 5-drug combination antiretroviral therapy. PLoS One. 2011; 6:e20967. [PubMed: 21738597]

14. Clifford DB, Nath A, Cinque P, et al. A study of mefloquine treatment for progressive multifocal leukoencephalopathy: results and exploration of predictors of PML outcomes. J Neurovirol. 2013; 19:351-8. [PubMed: 23733308]

15. Vermersch PD, Kappos LM, Gold RM, et al. Clinical outcomes of natalizumab-associated progressive multifocal leukoencephalopathy. Neurology. 2011; 76:1697-704. [PubMed: 21576685]

16. Huang D, Cossoy M, Li M, et al. Inflammatory progressive multifocal leukoencephalopathy in human immunodeficiency virus-negative patients. AnnNeurol. 2007; 62:34-9.

17. Martin-Blondel G, Bauer J, Cuvinciuc V, et al. In situ evidence of JC virus control by CD8+ T cells in PML-IRIS during HIV infection. Neurology. 2013; 81:964-70. **Pathologic study substantiating characteristics of cellular response in IRIS compared to non inflammatory PML cases. [PubMed: 23935178]

18. Metz I, Radue EW, Oterino A, et al. Pathology of immune reconstitution inflammatory syndrome in multiple sclerosis with natalizumab-associated progressive multifocal leukoencephalopathy. Acta Neuropathol. 2012; 123:235-45. [PubMed: 22057786]

19. Wuthrich C, Popescu BF, Gheuens S, et al. Natalizumab-associated progressive multifocal leukoencephalopathy in a patient with multiple sclerosis: a postmortem study. J Neuropathol Exp Neurol. 2013; 72:1043-51. [PubMed: 24128680] 
20. Schippling S, Kempf C, Buchele F, et al. JC virus granule cell neuronopathy and GCN-IRIS under natalizumab treatment. Ann Neurol. 2013; 74:622-6. [PubMed: 23868420]

21. Dang X, Vidal JE, Oliveira AC, et al. JC virus granule cell neuronopathy is associated with VP1 C terminus mutants. The Journal of general virology. 2012; 93:175-83. [PubMed: 21940415]

22. Dang L, Dang X, Koralnik IJ, Todd PK. JC Polyomavirus Granule Cell Neuronopathy in a Patient Treated With Rituximab. JAMA neurology. 2014; 71:487-9. [PubMed: 24515530]

23. Lach B, Connolly B, Wuthrich C, Koralnik IJ. Inflammatory infratentorial progressive multifocal leukoencephalopathy in a patient with rheumatoid arthritis. Neuropathology. 2014; 34:39-44. [PubMed: 23683127]

24. Yousry TA, Pelletier D, Cadavid D, et al. Magnetic resonance imaging pattern in natalizumabassociated progressive multifocal leukoencephalopathy. Ann Neurol. 2012; 72:779-87. [PubMed: 23280794]

25. Wattjes MP, Richert ND, Killestein J, et al. The chameleon of neuroinflammation: magnetic resonance imaging characteristics of natalizumab-associated progressive multifocal leukoencephalopathy. Mult Scler. 2013; 19:1826-40. *Excellent review of imaging characteristics of PML in setting of natalizumab with reference to IRIS. [PubMed: 24192217]

26. Wattjes MP, Verhoeff L, Zentjens W, et al. Punctate lesion pattern suggestive of perivascular inflammation in acute natalizumab-associated progressive multifocal leukoencephalopathy: productive JC virus infection or preclinical PML-IRIS manifestation? J Neurol Neurosurg Psychiatry. 2013; 84:1176-7. [PubMed: 23695498]

27. Khoury MN, Alsop DC, Agnihotri SP, et al. Hyperintense cortical signal on magnetic resonance imaging reflects focal leukocortical encephalitis and seizure risk in progressive multifocal leukoencephalopathy. Ann Neurol. 2014; 75:659-69. *Characterization of imaging, pathologic and clinical associations relevant to PML IRIS. [PubMed: 24752885]

28. Gheuens S, Smith DR, Wang X, Alsop DC, Lenkinski RE, Koralnik IJ. Simultaneous PML-IRIS after discontinuation of natalizumab in a patient with MS. Neurology. 2012; 78:1390-3. [PubMed: 22517104]

29. Gheuens S, Ngo L, Wang X, Alsop DC, Lenkinski RE, Koralnik IJ. Metabolic profile of PML lesions in patients with and without IRIS: an observational study. Neurology. 2012; 79:1041-8. [PubMed: 22914832]

30. Dahlhaus S, Hoepner R, Chan A, et al. Disease course and outcome of 15 monocentrically treated natalizumab-associated progressive multifocal leukoencephalopathy patients. J Neurol Neurosurg Psychiatry. 2013; 84:1068-74. [PubMed: 23606731]

31. Dong-Si T, Richman S, Wattjes MP, et al. Outcome and survival of asymptomatic PML in natalizumab-treated MS patients. Annals of clinical and translational neurology. 2014; 1:755-64. [PubMed: 25493267]

32. Khatri B, Fox R, Koo A, et al. The effect of plasma exchange in accelerating clearance of natalizumab in patients with multiple sclerosis: results of the PLEX study (poster presented at 23rd Congress of the European Committee for Treatment and Research in Multiple Scerosis ECTRIMS). Multiple Sclerosis. 2007; 13:S172.

33. Antoniol C, Jilek S, Schluep M, et al. Impairment of JCV-specific T-cell response by corticotherapy: effect on PML-IRIS management? Neurology. 2012; 79:2258-64. [PubMed: 23175722]

34. Giacomini PS, Rozenberg A, Metz I, et al. Maraviroc and JC virus-associated immune reconstitution inflammatory syndrome. The New England journal of medicine. 2014; 370:486-8. [PubMed: 24476450]

35. Sospedra M, Schippling S, Yousef S, et al. Treating Progressive Multifocal Leukoencephalopathy With Interleukin 7 and Vaccination With JC Virus Capsid Protein VP1. Clinical infectious diseases : an official publication of the Infectious Diseases Society of America. 2014; 59:1588-92. [PubMed: 25214510]

36. Boulware DR, Rolfes MA, Rajasingham R, et al. Multisite validation of cryptococcal antigen lateral flow assay and quantification by laser thermal contrast. Emerging infectious diseases. 2014; 20:45-53. **Important study showing delay of cART is important to outcomes when initiating therapy for cryptococcal meningitis in HIV therapy naïve individuals. This finding differs from 
recommendations for other HIV associated OI probably because of the impact of IRIS. [PubMed: 24378231]

37. McKenney J, Smith RM, Chiller TM, et al. Prevalence and correlates of cryptococcal antigen positivity among AIDS patients--United States, 1986-2012. MMWR Morbidity and mortality weekly report. 2014; 63:585-7. [PubMed: 25006824]

38. Jarvis JN, Govender N, Chiller T, et al. Cryptococcal antigen screening and preemptive therapy in patients initiating antiretroviral therapy in resource-limited settings: a proposed algorithm for clinical implementation. Journal of the International Association of Physicians in AIDS Care. 2012; 11:374-9. [PubMed: 23015379]

39. Meyer AC, Kendi CK, Penner JA, et al. The impact of routine cryptococcal antigen screening on survival among HIV-infected individuals with advanced immunosuppression in Kenya. Tropical medicine \& international health : TM \& IH. 2013; 18:495-503. [PubMed: 23368667]

40. Vijayan T, Chiller T, Klausner JD. Sensitivity and specificity of a new cryptococcal antigen lateral flow assay in serum and cerebrospinal fluid. MLO: medical laboratory observer. 2013; 45:16, 8 . 20. [PubMed: 23822028]

41. Alemu AS, Kempker RR, Tenna A, et al. High prevalence of Cryptococcal antigenemia among HIV-infected patients receiving antiretroviral therapy in Ethiopia. PLoS One. 2013; 8:e58377. [PubMed: 23469276]

42. Gates-Hollingsworth MA, Kozel TR. Serotype sensitivity of a lateral flow immunoassay for cryptococcal antigen. Clinical and vaccine immunology : CVI. 2013; 20:634-5. [PubMed: 23365202]

43. Rugemalila J, Maro VP, Kapanda G, Ndaro AJ, Jarvis JN. Cryptococcal antigen prevalence in HIV-infected Tanzanians: a cross-sectional study and evaluation of a point-of-care lateral flow assay. Tropical medicine \& international health : TM \& IH. 2013; 18:1075-9. [PubMed: 23937699]

44. Escandon P, Lizarazo J, Agudelo CI, Chiller T, Castaneda E. Evaluation of a rapid lateral flow immunoassay for the detection of cryptococcal antigen for the early diagnosis of cryptococcosis in HIV patients in Colombia. Medical mycology : official publication of the International Society for Human and Animal Mycology. 2013; 51:765-8.

45. Chang CC, Lim A, Omarjee S, et al. Cryptococcosis-IRIS is associated with lower cryptococcusspecific IFN-gamma responses before antiretroviral therapy but not higher T-cell responses during therapy. J Infect Dis. 2013; 208:898-906. *Predictive findings for cryptococcal IRIS. [PubMed: 23766525]

46. Boulware DR, Bonham SC, Meya DB, et al. Paucity of initial cerebrospinal fluid inflammation in cryptococcal meningitis is associated with subsequent immune reconstitution inflammatory syndrome. Journal of Infectious Diseases. 2010; 202:962-70. [PubMed: 20677939]

47. Robertson EJ, Najjuka G, Rolfes MA, et al. Cryptococcus neoformans ex vivo capsule size is associated with intracranial pressure and host immune response in HIV-associated cryptococcal meningitis. J Infect Dis. 2014; 209:74-82. [PubMed: 23945372]

48. Haynes BC, Skowyra ML, Spencer SJ, et al. Toward an integrated model of capsule regulation in Cryptococcus neoformans. PLoS Pathog. 2011; 7:e1002411. [PubMed: 22174677]

49. Longley N, Harrison TS, Jarvis JN. Cryptococcal immune reconstitution inflammatory syndrome. Current opinion in infectious diseases. 2013; 26:26-34. [PubMed: 23242412]

50. Manji H, Jager HR, Winston A. HIV, dementia and antiretroviral drugs: 30 years of an epidemic. J Neurol Neurosurg Psychiatry. 2013; 84:1126-37. [PubMed: 23378642]

51. Deeks SG, Tracy R, Douek DC. Systemic Effects of Inflammation on Health during Chronic HIV Infection. Immunity. 2013; 39:633-45. [PubMed: 24138880]

52. Venkataramana A, Pardo CA, McArthur JC, et al. Immune reconstitution inflammatory syndrome in the CNS of HIV-infected patients. Neurology. 2006; 67:383-8. [PubMed: 16894096]

53. Roh D, Glenn MD, Petito CK, Post MJ, Verma A. Reversible severe encephalitis and word deafness following rapid immune reconstitution in AIDS: a case report. J Neurovirol. 2013; 19:190-3. [PubMed: 23580250]

54. Costello DJ, Gonzalez RG, Frosch MP. Case 18-2011: A 35-year-old HIV-positive woman with headache and altered mental status. NEnglJMed. 2011; 364:2343-52. 
55. Gray F, Lescure FX, Adle-Biassette H, et al. Encephalitis with Infiltration by CD8+ Lymphocytes in HIV Patients Receiving Combination Antiretroviral Treatment. Brain Pathol. 2013; 23:525-33. **Fascinating description of unique CD8+ cell associated encephalitis during treatment of HIV. This likely represents a unique form of IRIS from HIV. [PubMed: 23347174]

56. Johnson TP, Patel K, Johnson KR, et al. Induction of IL-17 and nonclassical T-cell activation by HIV-Tat protein. Proc Natl Acad Sci U S A. 2013; 110:13588-93. [PubMed: 23898208] 


\section{Key points}

- Damaging IRIS is worst after advanced immunodeficiency in the setting of a severe underlying opportunistic infections such as PML and cryptococcal meningitis

- Early diagnosis and treatment of HIV or its complications can yield optimal clinical outcomes

- Presymptomatic diagnosis of PML by close monitoring of high risk individuals, can minimize neurological disability and morbidity

- Corticosteroid treatment of IRIS in PML has led to better outcomes than previously observed, but must be judiciously applied due to theoretical risk of impairing immune responses needed to control the infection

- Cryptococcal meningitis treatment should be established prior to initiation of cART in HIV due to inferior survival when early cART is used 

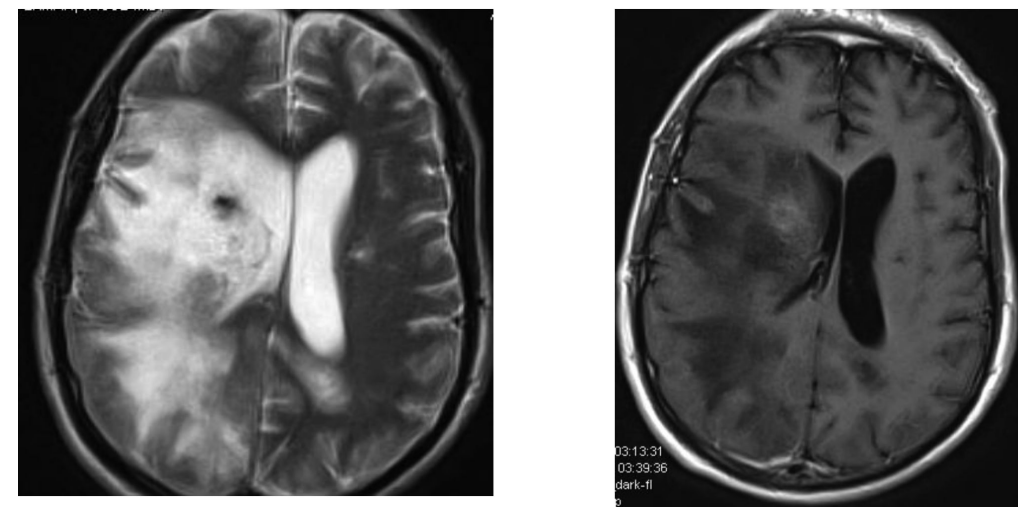

Figure 1.

Late consequence of HIV associated PML, seven months after PML diagnosis and initiation of cART. Left image is T2 brain MRI showing extensive white matter changes from PML as well as residual mass effect from resolving PML IRIS. Right image shows remaining gadolinium enhancement in the lesion from resolving IRIS. The image emphasizes the long duration of inflammation present in lesions of PML with inflammatory changes. The patient has suffered recurrent seizures, also frequently associated with inflammatory PML. 